\title{
Objective Metrics for Functional Evaluation of Upper Limb during the ADL of Drinking: Application in SCI
}

\author{
A. de los Reyes-Guzmán, I. Dimbwadyo-Terrer, S. Pérez-Nombela , F. Trincado \\ D. Torricelli, and A. Gil-Agudo
}

\begin{abstract}
Three-dimensional kinematic analysis provides quantitative assessment of upper limb motion and is used as an outcome measure to evaluate movement disorders. The aim of the present study is to present a set of kinematic metrics for quantifying characteristics of movement performance and the functional status of the subject during the execution of the activity of daily living (ADL) of drinking from a glass. Then, the objective is to apply these metrics in healthy people and a population with cervical spinal cord injury (SCI), and to analyze the metrics ability to discriminate between healthy and pathologic people. 19 people participated in the study: 7 subjects with metameric level $\mathrm{C} 6$ tetraplegia, 4 subjects with metameric level $\mathrm{C} 7$ tetraplegia and 8 healthy subjects. The movement was recorded with a photogrammetry system. The ADL of drinking was divided into a series of clearly identifiable phases to facilitate analysis. Metrics describing the time of the reaching phase, the range of motion of the joints analyzed, and characteristics of movement performance such as the efficiency, accuracy and smoothness of the distal segment and inter-joint coordination were obtained. The performance of the drinking task was more variable in people with SCI compared to the control group in relation to the metrics measured. Reaching time was longer in SCI groups. The proposed metrics showed capability to discriminate between healthy and pathologic people. Relative deficits in efficiency were larger in SCI people than in controls. These metrics can provide useful information in a clinical setting about the quality of the movement performed by healthy and SCI people during functional activities.
\end{abstract}

Keywords-Metrics, Kinematic, Assessment, Upper limb, Activities of Daily Living.

\section{INTRODUCTION}

The upper limb function is affected in more than $50 \%$ of spinal cord injuries [1].

Impairment and disability in clinical settings is generally assessed by ordinal scales such as the Fugl-Meyer Assessment, Frenchay Arm Test, Motor Assessment Scale, Box and Block Test, and the Nine-Hole Peg Test [2], but they may not be sensitive to small and more specific changes [3]. It's important that measures have the ability to detect a minimal clinically important change in subjects. At the same time, the measures must have the ability to give consistent responses on repeated assessments in the absence of change in the characteristic being studied [4]. Moreover, the use of these scales is not exempt from a degree of subjectivity. So more objective assessment methods are needed to evaluate and describe the upper limb function in detail.

On the other hand kinematic movement analysis can provide more specific information about movement component and strategies, although this requires special equipment. Kinematics describes movements of the body through space and time without reference to the forces involved [5]. It's of special importance to translate data from kinematic analysis in terms of objective metrics with a useful sense and easily understood in a clinical setting. These metrics can synthetize the main aspects of the movement that directly relate with functional ability

The aim of this study is to present a set of objective metrics for the functional upper limb evaluation and for the measure characteristics of quality movement during functional activities. Then these metrics are applied to a population of healthy and cervical SCI people during the ADL of drinking from a glass. The last objective is to analyze the ability of these metrics as discriminative measures between healthy people and people with SCI.

\section{Materials AND MEthods}

\section{A. Population}

Nineteen subjects participated in this study and were separated into three groups: a control group (CG) with 8 subjects; 7 people with $\mathrm{C} 6$ tetraplegia (C6 group) and 4 people with $\mathrm{C} 7$ tetraplegia (C7 group). The demographic and anthropometric characteristics are shown in Table 1. In the case of subjects with $\mathrm{C} 6$ or $\mathrm{C} 7$ tetraplegia, the etiology of the injury was trauma in every case. The patients screened had to fulfill the following criteria to be included in the study: age 16 to 65 years, injury of at least 6 months' duration and level of injury $\mathrm{C} 6$ or $\mathrm{C} 7$ classified according to the American Spinal Cord Injury Association (ASIA) [6] scales into grades A or B. The subjects were classified into 
$\mathrm{C} 6$ or $\mathrm{C} 7$ tetraplegia by a physical examination. The upper limb Motor Index was obtained [6].

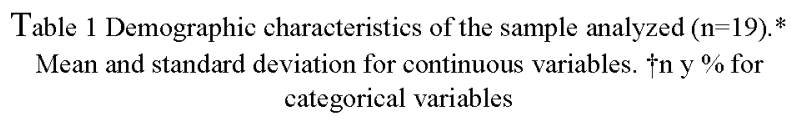

Table 1 Demographic characteristics of the sample analyzed $(n=19)$.* Mean and standard deviation for continuous variables. $\nmid \mathrm{n}$ y $\%$ for categorical variables

\begin{tabular}{lccc}
\hline \multicolumn{1}{c}{ Item } & CG & C6 & C7 \\
\hline Sex (male) & $3(37.5)$ & $4(57.4)$ & $4(100)$ \\
Age (years) & $26.0(5.0)$ & $34.0(5.0)$ & $30.5(10.0)$ \\
Height (cm) & $168.0(20.0)$ & $175.0(10.0)$ & $184.0(10.0)$ \\
Weight (Kg) & $65.0(21.1)$ & $90.2(7.1)$ & $79.0(9.1)$ \\
Months since injury & - & $8.5(2.2)$ & $7.5(1.8)$ \\
ASIA (A) & - & $3(33.3)$ & $2(50)$ \\
ASIA (B) & - & $4(66.6)$ & $2(50)$ \\
Index Motor right arm & $25.0(0)$ & $13.0(3.0)$ & $14.5(2.0)$ \\
\hline
\end{tabular}

All patients signed an informed consent form before the study. The guidelines of the declaration of Helsinki were followed in every case and the study design was approved by the local ethics committee.

\section{B. Experimental Set-Up and Data Processing}

Three-dimensional movement capture was recorded with the photogrammetry equipment CodaMotion (Charnwood Dynamics, Ltd, UK) following the protocol in an earlier study [7].

All the subjects were right handed and performed the movement of the ADL of drinking with the right arm at a self-selected speed. Subjects with C6 or C7 tetraplegia sat in their own wheelchairs and the controls sat in a conventional wheelchair. The movement was performed in front of a table, from a standardized position. The complete experimental protocol was included in a previous study, in which the consistency and repeatability of the experimental setup was assessed. The recordings were processed with Visual 3D software (C-Motion, Inc., USA) [7].

The ADL of drinking from a glass was divided into a series of clearly identifiable phases to facilitate analysis. We used phases and events delimiting the phases that have been described previously: reaching, forward transport, drinking, back transport and return [7].

\section{Kinematic Metrics Definition}

A set of objective kinematic metrics was defined from experimental data preprocessed with Visual3D software. These metrics were separated into two groups and all of them were calculated in Matlab software: a) Metrics in Relation to Spatio-temporal Variables from Preprocessed Data with Visual3D:

Reaching time ( $T_{\text {Reaching }}$ ): duration of the reaching phase normalized by the duration of the complete cycle (Tcycle) expressed in percentage.

$$
T_{\text {Reaching }}=\left(\frac{T_{\text {Reaching }}}{T_{\text {Cycle }}}\right) 100
$$

Range of motion (ROM): this metric is defined as the sum of the ROM in degrees for each of the six degrees of freedom (DoF), normalized by the sum of the total biologically allowed ROM for each DoF analyzed [8]. So the metric is obtained dimensionless expressed as a percentage. The ROM is defined here as the difference between the maximum and minimum angles recorded by a given joint over the course of the drinking task.

$$
R O M_{\text {Total }}=\frac{\sum_{i=1}^{N}\left(\theta_{\max }-\theta_{\min }\right)_{\text {subject }}}{\sum_{i=1}^{N}\left(\theta_{\max }-\theta_{\min }\right)_{\text {total }}} * 100
$$

\section{b) Metrics for the Evaluation of Functional Abilities and Skills of the Upper Limb}

These parameters are defined from the kinematic of the distal segment, the hand, and they are measurements of the quality of the movement performed by the three groups of people. These metrics are:

Efficiency (E): the movement efficiency is obtained by computing the path length $\left(\mathrm{L}_{\text {path }}\right)$ of the trajectory traveled by the subject to reach the target (in this study the glass used to perform the ADL of drinking from a glass) $[9,10]$. This parameter is obtained by summing the distances between two consecutive points of the subject's path; then, it's normalized to the straight line distance between the starting point of the task and the target $\left(d_{s 1}\right)$. A ratio between $L_{\text {path }}$ and $\mathrm{d}_{\mathrm{s} 1}$ of 1 represents a direct movement to the target (ideal), and a reach path ratio slightly higher than 1 represents a healthy movement but a value proximal to 2 an abnormally curved movement. This ratio is calculated during the reaching phase of the task, and it is a measure of the error of movement efficiency, so a transformation function has been applied with the aim of higher values (maximum 100) correspond to a more efficient movement (equation 3).

$$
E=130-30 *\left(\frac{L_{p a t h}}{d_{s l}}\right)
$$


Accuracy (A): this metric is assessed by measuring the mean absolute value of the distance $\left(\mathrm{d}_{\text {mean }}\right)$ of each point of the path from the theorical path (the straight line between initial point and the target) [9] and the maximal distance $\left(d_{\max }\right)$. This result is inverted with the aim of higher values correspond to more accuracy movements.

$$
A=100-\left(d_{\text {max }}+d_{\text {mean }}\right)
$$

Smoothness (S): is defined from the number of peaks in the hand speed profile during the reaching and proximal transport phases [5]. To avoid signal noise effects, the time between two subsequent peaks had to be at least 150 milliseconds. Fewer peaks in speed represent fewer periods of acceleration and deceleration, making a smoother movement. For that reason, the smoothness metric has been defined as the inverse of speed peaks number (equation 5), so higher values of smoothness metric correspond to smoother movements and a better functional state.

$$
S=20-\text { npeaks }
$$

Coordination $(C)$ : the inter-joint coordination between the shoulder and elbow joint angles for the flexionextension movement was characterized quantitatively by means of the Pearson correlation index [7], as expressed the following equation. This metric is obtained from covariance matrix and marginal deviations between both variables analyzed (Equation 6). A correlation index closer to 1.0 indicates stronger correlation and indicates that the joint motion of the 2 joints is tightly coupled. Then, this value is multiplied by a 100 factor for obtaining a coordination value between 0 and 100 points.

$$
C=\frac{\frac{\sum F_{S} F_{E}}{N}-\overline{F_{S} F_{E}}}{S_{F_{S}} S_{F_{E}}} * 100
$$

\section{Statistical Analysis}

A descriptive analysis with SPSS for Windows (version 12.0) was made of the clinical and functional variables (Table 1). The Kruskal-Wallis test was used to find possible differences in each metric between the three groups analyzed. The Bonferroni correction was applied, which takes into account randomness due to multiple comparisons.

The repeatability of the protocol was evaluated in an earlier study [7].

\section{RESUlts}

There were no significant differences in demographic variables between participants with SCI and healthy people. The most relevant results are shown in Table 2.

The range of motion (ROM), expressed as percentage, is obtained by summing the ROM of each joint analyzed and then normalized by the total theoretically allowed.

In relation to the movement time, people with $\mathrm{C} 6$ tetraplegia had slower movement times than healthy people in reaching phase normalized by the duration of the complete cycle $(p<0.01)$.

\begin{tabular}{|c|c|c|c|}
\hline METRICS & $\mathrm{CG}(\mathrm{n}=8)$ & $\mathrm{C} 6(\mathrm{n}=7)$ & $\mathrm{C} 7(\mathrm{n}=4)$ \\
\hline Range of motion (\%) & $24.52(1.19)^{a, b}$ & $28.84(4.80)^{a}$ & $30.18(5.50)^{\mathrm{b}}$ \\
\hline Reaching time $(\%)$ & $14.10(3.74)^{\mathrm{c}}$ & $22.61(15.52)^{c}$ & $14.58(4.14)$ \\
\hline Efficiency (\%) & $97.81(2.04)^{c, d}$ & $77.27(32.44)^{c}$ & $93.16(8.83)^{d}$ \\
\hline Accuracy (points) & $87.69(5.86)^{a}$ & $80.01(7.59)^{a}$ & $86.61(4.12)$ \\
\hline $\begin{array}{l}\text { Smoothness } \\
\quad \text { (inverse peaks number) }\end{array}$ & $18.00(0.25)^{c, d}$ & $15.00(2.50)^{c}$ & $16.50(1.00)^{d}$ \\
\hline Coordination (\%) & $88.42(6.98)$ & $69.88(22.89)$ & $82.00(6.15)$ \\
\hline
\end{tabular}

Table 2 Median and interquartile range of the metrics for the three groups analyzed.

Same letters mean significant differences between groups $\mathrm{a}, \mathrm{b}: \mathrm{p}<0.05$ with Bonferroni correction c,d: $\mathrm{p}<0.01$ with Bonferroni correction

The metrics efficiency and accuracy were calculated from the hand path during the reaching phase within the $\mathrm{ADL}$ of drinking from a glass. The movement was accurate in $\mathrm{C} 7$ group as in the healthy people, but less efficient $(\mathrm{p}<0.01)$. The movement was less accurate $(\mathrm{p}<0.05)$ and efficient $(\mathrm{p}<0.01)$ in C6 group that in the controls (Figure 1) (Table 2).

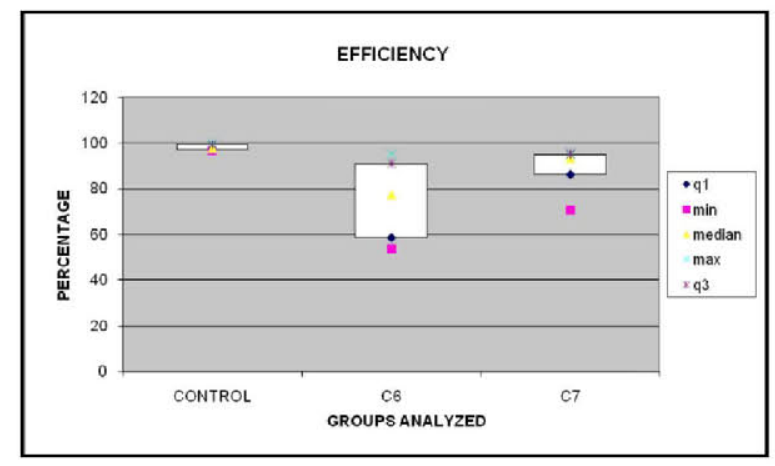

Fig. 1 Results of the efficiency metric for the three groups analyzed 
Smoothness was obtained during the reaching and during the transport of the glass toward the mouth, so the minimum number of peaks in velocity profile is 2 . Then the result of the three metrics is inverted for expressing it, as a greater value correspond to a better functional state. Statistically significant differences were found between healthy and C6 subjects for the metric efficiency and smoothness ( $p<0.01$ ), and for the metric accuracy $(p<0.05)$.

In relation to the inter-joint coordination, no statistically significant differences were found (Table 2).

In the C6 group, there was a great variability in the measured results for the efficiency (Figure 1) and coordination metrics (Table 2).

\section{DISCUSSION}

The metrics proposed can synthetize the main aspects of the movement that directly relate with functional capability. The main definition of the most of the metrics proposed in this study can be found in literature $[9,10]$. The inverse functions have been developed within this study with the aim of obtaining the results according to functional clinical tests.

It is worth noting that these parameters may provide useful information related to the quantitative data we can obtain, in situations in which, despite a high value of movement accuracy, the subject performs a longer path with the hand toward the target, and the consequence is a less efficient movement. The smoothness, efficiency and range of movement showed the strongest ability to discriminate movement quality between healthy and SCI people. This is confirmed, for smoothness metric, by Murphy et al. in a study in stroke people [5]. In this study, we calculated speed peaks during reaching and forward transport phases within the ADL of drinking because these two phases correspond to two different constraints of a purposeful movement.

The reaching phase, including grasping, it's the movement phase of the ADL of drinking from a glass with greater contribution to the total movement time $[5,7]$.

On the other hand, kinematic analyses are increasingly being used in clinical research as evaluative measures [5]. It's necessary additional assessment to investigate the relation between kinematics and clinical scales. Moreover it is important to take into account that the analysis of a purposeful daily activity increases the validity of the study.

\section{CONCLUSIONS}

In the present study, the metrics proposed were applied to a population with SCI people, and their ability to discriminate between healthy and SCI people, has been shown.
For further studies the sample analyzed will be greater and the metrics will be applied to other neurological pathologies such as stroke, where the discriminative ability within this pathology will be investigated.

\section{ACKNOWLEDGMENT}

Fundings were obtained from the research project entitled "HYPER: Dispositivos Híbridos Neuroprotésicos y Neurorobóticos para Compensación Funcional y Rehabilitación de Trastornos del Movimiento". Referencia: CSD00C09-61313. CONSOLIDER-INGENIO 2010. Ministerio de Ciencia e Innovación.

\section{REFERENCES}

1. Wyndaele M, Wyndaele JJ (2006). Incidence, prevalence and epidemiology of spinal cord injury: what learns a worldwide literature survey?. Spinal Cord 21(8): 357-364

2. Wade DT (1992) Measurement in neurological rehabilitation. Oxford medical publications. Oxford University Press, 388

3. Mc Crea PH, Eng JJ, Hodgson AJ (2002) Biomechanics of reaching: clinical implications for individuals with acquired brain injury. Disabil Rehabil, 24: 534-541

4. Boyce W, Gowland C, Rosenbaum P et al. (1991) Measuring quality of movement in cerebral palsy: a review of instruments. Phys Ther. 71: 813-819

5. Murphy M, Willén C, Sunnerhagen K (2011) Kinematic variables quantifying upper-extremity performance after stroke during reaching and drinking from a glass. Neurorehabil Neural Repair 25:71 DOI $10.1177 / 1545968310370748$

6. Maynard FM, Bracken MB, Creasey G et al. (1997) Standards for Neurological and Functional Classification of Spinal Cord Injury. American Spinal Injury Association. Spinal Cord, 35: 266-274

7. Ana de los Reyes-Guzmán, Angel Gil-Agudo, Benito PeñascoMartín, Marta Solís-Mozos, Antonio del Ama-enEspinosa, Enrique Pérez-Rizo (2010) Kinematic analysis of the daily activity of drinking from a glass in a population with cervical spinal cord injury. http://www.jneuroengrehab.com/content $/ 7 / 1 / 41$

8. Kapandji AI (2012) Fisiología articular. Miembro superior. $6^{a}$ Edition. Editorial Medica Panamericana

9. Colombo R, Pisano F, Micera $S$ et al (2008) Assessing mechanisms of recovery during robot-aided neurorehabilitation of the upper limb. Neurorehabil Neural Repair 22:50 DOI 10.1177/1545968307303401

10. Lang CE, Wagner JM, Bastian AJ et al (2005) Deficits in grasp versus reach during acute hemiparesis. Exp Brain Res 166: 126-136 DOI $10.1007 / \mathrm{s} 00221-005-2350-6$

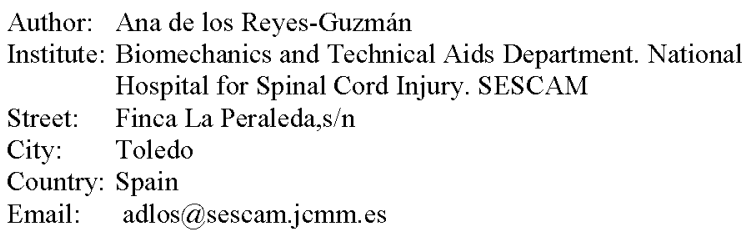

